\title{
Comparative evaluation of adhesive performance to dentin: study in patients of different ages
}

\author{
J.V. Mandra ${ }^{*}$, S.L. Votyakov ${ }^{2}$, M.E. Smirnova ${ }^{1}$, S.E. Zholudev ${ }^{3}$, and S.S.Grigorjev ${ }^{1}$ \\ ${ }^{1}$ Ural State Medical University, Department of Therapeutic Dentistry and Propaedeutics of Dental \\ Diseases, 620028, Repina street, 3, Ekaterinburg, Russian Federation \\ ${ }^{2}$ Institute of Geology and Geochemistry, Ural Branch, Russian Academy of Science, 620016, \\ Akademika Vonsovskogo street, 15, Ekaterinburg, Russian Federation \\ ${ }^{3}$ Ural State Medical University, Department of Orthopedic and General Practice Dentistry, 620028, \\ Repina street, 3, Ekaterinburg, Russian Federation
}

\begin{abstract}
The article describes in vitro evaluation of the micro-shear bond strength between composite resin and dentin specimens treated with self-etching, total-etching and universal dental adhesives for patients of different ages. The authors compared microstructure of resin-infiltrated zone for self-etching, total-etching and universal dental adhesive systems by means of scanning electron microscopy. These findings presented negatively effects of aging on the bond strength of dentinal surfaces and the advantages of adhesive protocol with universal adhesives for all ages.
\end{abstract}

\section{Introduction}

The proportion of older people continues to grow worldwide, especially in developing countries. The negative impact of poor oral conditions on the quality of life of older adults is an important public health issue [1]. Aging is the physiologic change that occurs over time and at different ways, that are related to lifestyle, environment and genetics. It can be a challenge to differentiate normal aging of the oral cavity from disease [2].

With increasing age the teeth demonstrate wearing of the enamel, chipping and fracture lines, darker color. The pulp chamber and canals are reduced in size as a result of the secondary dentin deposition [1, 2, 3]. However, coronal or root caries is the most represented disease of patients over 65 years old (100\%), though the peak incidence being observed at 18-30 years of age. Periodontal attachment loss also occurs in association with aging, usually manifests as periodontitis or recession on the buccal surface of teeth, occurs in $89 \%$ of the population over 65 years old, but the peak incidence is observed at 3540 years $[4,5]$. Oral mucosa changes with aging include reduced wound-healing capacity [6]. However, environmental factors, such as smoking, vaping, occupational hazards dramatically increase the risk of mucosal pathology $[2,3]$. Reduced salivary gland function is often seen in association with medication usage, as well as with disorders such as

*Corresponding author: jmandra@mail.ru 
diabetes, cardiovascular problems, etc. Both medication use and chronic diseases are more common in older adults $[4,5]$. Masticatory function is of particular importance for aging, and nutritionally complete diet is necessary for avoiding sarcopenia and the frailty syndrome. Successful oral aging is associated with adequate function and comfort. A reduced, but functional occlusion with implants or dentures can be proposed as a measure of successful oral aging. Healthy oral aging is important to whole process of healthy aging from both biological and social perspectives $[2,4,5,6]$.

Although tooth enamel is the hardest tissue with the highest mineral content in human organism the following age-related changes occur: flatting cusps and incisal edges, worn microstructural details, loss of perikymata. Yet the enamel surface shows reduced shine, elastic modulus and permeability with age, but both hardness and strength can increase over $12 \%$ by age 55 and over. Consequently the brittleness also is growing and cracks are often in enamel aging $[2,7]$.

The main changes of dentin aging assotiate with an increasing number of dead tracts, thickness and vitality, obturation of dentin tubules, which become fully occluded by age 7580. Dentin undergoes important structural change - the formation of sclerotic, reparative and reactive dentin. It is well known that the aging changes chemical and surface structure of tooth tissues. Age changes in tooth hard tissues states increasing of fluoride, decreasing of water and organic content [2]. They can significantly depend on lifestyle of a particular person, environmental conditions, $\mathrm{pH}$ levels of foods and beverages, hereditary factors, etc. Relating to the same age group patients may have a different state of the appearance, composition, resiliency and strength of teeth and surrounding tissues [2, 4]. Although changes in apperance and structure of the teeth with aging, they can remain functional for all lifetime with proper care and adequate restoration. Adhesive technologies and invasive methods can be used for direct and indirect restoration of caries and non-caries lesions. The main principe of tooth bonding is associated with micromechanical retention by mean of different adhesives [7].

The oral cavity is an accessible area for study of aging changers during the routine treatment of tooth deseases. Several reports have indicated that resin bond strengths to sclerotic noncarious cervical dentin lower than to normal dentin. This fact is explained by the tubule occlusion with mineral salts, preventing resin tag formation. Research with transmission electron microscopy revealed that in addition to occlusion of the tubules by mineral crystals, sclerotic dentin of wedge-shaped cervical lesions contains a hypermineralised surface that resists the etching action. This layer prevents hybridisation of the sclerotic dentine [8].

It was found that adhesives containing different structures/concentrations of functional monomers produced interfaces with obviously different chemical and morphological characteristics. These findings support the concept that the stable chemical bonding produced by 10-MDP (10-methacryloyloxydecyl dihydrogen phosphate) to hydroxyapatite is advantageous for durability of adhesive-dentin bonds. In contrast a higher immediate bond strength was achieved with the functional monomer GPDM (glycero-phosphate dimethacrylate). That's why, self-etch and total-etch adhesives with monomers 10-MDP and GPDM may have important impact on bonding effectiveness [9].

New multi-mode universal adhesives offer clinicians the choice of using the etch-andrinse technique, selective enamel etch technique or self-etch technique for bonding to tooth substrates. The studies examined the bonding of five universal adhesives to human coronal dentine and demonstrated the increase in versatility without compromising its adhesive effectiveness. [10]. The shear bond strength of metal brackets bonded with different universal adhesive systems containing 10-MDP and Self Etching Primer after 20,000 thermal cycles was evaluated. Statistical analysis showed that the universal adhesive systems presented mean shear bond strength values similar to self-etching primer [11]. The 
comparing study of the dentin bond strength with a new universal adhesive and contemporary multi-step total etch-adhesives revealed a homogenous adhesive layer formation. A new universal adhesive can make reliable bond to dentin, regardless of the application mode $[12,13]$. Adhesive bond strength plays an important role in determining the clinical performance and longevity of dental restorations for aged patients $[14,15]$. Nevertheless, bond strength tests for aging dentin has never been executed.

The purpose of the study was to compare the micro-shear bond strength between composite resin and dentin treated with self-etching, total-etching and universal dental adhesives in patients of various age groups (young people, the elderly and senile).

\section{Materials and Methods}

To study the micro-shear bond strength to dentin, 3 groups of patients were recruited in accordance with the WHO age classification. Group 1 consisted of patients of young age (18-44 years old, 20 people, men and women), group 2 - elderly patients (60-74 years, 20 people, men and women), group 3 - patients of senile age (75-90 years, 20 people, men and women).

Patients of the study groups underwent a comprehensive dental examination (interview, examination, additional research methods). Study was performed using 60 extracted intact human molars of these patients (20 of each group). The teeth, all extracted within a duration of 6 months, were kept in artificial saliva ( $0.9 \%$ sodium chloride solution) until the beginning of the experiment. The teeth were cleaned with non-fluoridated pumice and dental prophylactic cups. Each tooth was sectioned in a mesiodistal direction, inserted into an epoxy resin block, the upper portion of the crown dentin left unembedded. The cutting was proceeded at low speed with refrigeration. Subsequently, the specimens were randomly allocated into four groups (each containing 20 specimens): group 1 - SE (self etching with Adper Easy One, 3M - ESPE); group 2 - TE (total acid etching with Adper Single Bond, $3 \mathrm{M}$ - ESPE); group 3 - UT (total acid etching with Scotchbond Universal, 3M - ESPE); group 4 - US (selective etching with Scotchbond Universal, 3M - ESPE). Dentin surfaces treatment with acid etching in groups 2 and 3 included conditioning the surface by $37 \%$ phosphoric acid gel (3M-ESPE) for 15 seconds, followed by thorough water rinsing and air drying. Following surface treatment, a layer of adhesive was applied on the dentin surfaces using disposable brush tips and following the manufacturer's instructions. After gentle airdrying the specimens for 5 seconds, adhesive materials were polymerized for 20 seconds using a light-curing unit (Elipar S10, 3M). From each group, 1 sample was randomly selected to be analyzed with scanning electron microscopy (JSM6390LV, Jeol). The rest of the samples received composite resin materials (Bulk Fill Posterior A3, 3M-ESPE) on the prepared dentin surfaces. Prepared dentin surfaces in all groups were covered by composite. Composite resin material was manually applied in $3 \mathrm{~mm}$ plastic post on the surface of dentin and light-cured by a visible-light-curing unit for 30 seconds. The samples were stored in a physiological solution at body temperature. The micro-shear bond strength of the samples was tested on a tester machine (Shear Bond Tester, BISICO).

Statistical data processing was carried out in the software product «Statistica». To compare the average values of the indices of the independent groups, statistical analysis by one-way analysis of variance (ANOVA) and the nonparametric Mann-Whitney test was used. Differences in indicators were taken as significant at $\mathrm{p}<0.05$.

\section{Results and Discussion}


As a result, the lowest average micro-shear bond strength of the dentin samples was in group SE: $21.16 \pm 2.15,16.71 \pm 1.26,13.97 \pm 1.34(\mathrm{P}<0.05)$; the highest values dentin bond strength were in group UT: $33.39 \pm 1.13,30.26 \pm 0.76,26.35 \pm 1.34(\mathrm{P}<0.05)$; very close values to group UT of dentin bond strengths were registered in group US: $32.45 \pm 2.4$, $29.38 \pm 1.47,26.48 \pm 1.22 \quad(\mathrm{P}<0.05)$. A number of samples established bond strength decrease with aging, that reflected aging sclerotic changes in dentin. In particular, the minimal decrease of the micro-shear bond strength has been established in groups UT, US with aging of patients. The statistical analysis revealed that there was no statistically significant difference between the groups UT and US senile age (75-90 years) patients.

Table 1. The results of the micro-shear bond strength in patients of various ages.

\begin{tabular}{|c|c|c|c|}
\hline \multirow{2}{*}{ Signs } & \multicolumn{3}{|c|}{ Age } \\
\cline { 2 - 4 } & Young & Elderly & Senile \\
\hline \multirow{2}{*}{ SE } & $21.16(2.5 ; 2.1)$ & $\begin{array}{c}16.71(1.1 ; 1.4) \\
\mathrm{p}<0.05\end{array}$ & $\begin{array}{c}13.97(1.7 ; 1.2) \\
\mathrm{p}<0.05\end{array}$ \\
\hline \multirow{2}{*}{ TE } & $31.08(0,9 ; 1,5)$ & $\begin{array}{c}21.91(0,8 ; 1,35) \\
\mathrm{p}<0.05\end{array}$ & $\begin{array}{c}15,24(1,55 ; 0,95) \\
\mathrm{p}<0.05\end{array}$ \\
\hline \multirow{2}{*}{ UT } & $33.39(1,8 ; 2,0)$ & $\begin{array}{c}30.26(1,25 ; 1,8) \\
\mathrm{p}<0.05\end{array}$ & $\begin{array}{c}26,35(1,95 ; 1,08) \\
\mathrm{p}<0.05\end{array}$ \\
\hline \multirow{2}{*}{ US } & $32,45(1,1 ; 3,8)$ & $29,38(0,5 ; 2,1)$ & $\begin{array}{c}26,48(0,58 ; 1,9) \\
\mathrm{p}<0.05\end{array}$ \\
\hline
\end{tabular}

Note: in table $1 \mathrm{p}$ - in comparison with a group of young patients $(\mathrm{p}<0.05)$.

The dentin tubules were shown to be exposed and obturated by adhesive in SEM photographs of the dentin surfaces. Microstructural changes as well as surface roughening, induced in dentin by etchants and adhesives, increase the bonding surface area and the adhesion. 10- to 50-micron-thick resin-penetrated layer was observed at the interface between the dentin and resin. The values of the hybrid layers calculated by SEM photographs presented in table 2 .

Table 2. Thickness of hybrid layer in patients of various age groups.

\begin{tabular}{|c|c|c|c|}
\hline \multirow{2}{*}{ Dentin samples } & \multicolumn{3}{|c|}{ Age } \\
\cline { 2 - 4 } & Young & Elderly & Senile \\
\hline SE & 20 & 10 & 10 \\
\hline TE & 45 & 30 & 25 \\
\hline UT & 50 & 35 & 30 \\
\hline US & 30 & 25 & 25 \\
\hline
\end{tabular}

This study was conducted to compare age-related changes of bonding between dentin surfaces and composite resin with the application of different adhesive systems. The analysis of values of the micro-shear bond strength shows their decrease with age which seems quite logical and consistent with SEM data. Recent scanning electron microscopy revealed that in addition to occlusion of the tubules by mineral crystals, many parts of aged dentin contain a hypermineralised surface that resisted the etching action of both selfetching primers and phosphoric acid. Total etching the dentin surfaces proved to be more effective technique than self-etching in all groups.

At the same time universal adhesive systems exhibited higher bond strength than classic TE and SE techniques, and application of universal adhesives with total etching of dentine showed quite similar bond strength as selective etching. Our data reflect the occurrence of systemic processes in dentin especially in elderly and senior age, predominance of sclerosis and hypermineralisation, that's why general tendency of increasing adhesion by means of universal adhesives is proven fact due to the significant mechanism of chemical bonding. 
Chemical bonding occur the interaction of functional acid monomers (carboxylic groups, phosphonic or phosphate) with hydroxyapatite crystals in dentin. 10-MDP is an example of a functional monomer that can interact with calcium ions in hydroxyapatite crystals in a process that is conducted until the formation of calcium salts of MDP. Thereby universal adhesives are able to create an inter-diffusion zone similar to that created by acid etching and conditioning despite of age-related processes.

Thus, the results of this investigation report about the dentin/resin micro-shear bond strength evaluation with dental adhesives for patients of different ages identified significant manifestation of adhesion decrease as an age-dependent process, determined by physiological mechanisms of aging, described in world literature. However, our obtained data showed the practical advantage of universal adhesives because of relative stability to dentin aging changes and prevalence of chemical bonding mechanism.

\section{Conclusion}

Within the limitations of this in vitro study, it may be concluded that age-related processes of dentin negatively affect on the bond strength of composite resins. Contradictions still remain regarding the most effective way of adhesion. According to the results of the micro-shear bond strength evaluation and compared microstructure of resin-infiltrated zone after using self-etching, total-etching and universal dental adhesives, it might be suggested that a standard choice for practitioner can be defined for universal dental adhesives to aged patients. Probably the preference of universal adhesives to aged patients concerns not only the treatment of carious lesions, but non-carious defects too due to high bond strength, enough to permit quality retention of restoration.

\section{References}

1. I.B. Lamster, L. Asadourian, T. Del Carmen, P.K. Friedman, Periodontol 2000, 72(1), 96-107 (2016)

2. G.An, Geriartrics and Aging, 12(10), 503-517 (2009)

3. L.C. Niessen, D.J. Felele, Compend. Contin. Educ. Dent., 23(10), 4-11 (2002)

4. P.E. Petersen, D. Candelman, S. Arpin, H. Ogava, Community Dent. Health., 27(4), 257-267 (2010)

5. P.E. Petersen, T.Yamamoto, Community Dent. Oral. Epidemiol., 33(2), 81-92 (2006)

6. V.V. Bazarnyi, L.G. Polushina, A.Yu. Maksimova, E.N. Svetlakova, E.A. Sementsova, P.M. Nersesyan, Yu.V. Mandra, Clin. Lab. Diagn., 64(12), 736-740 (2019)

7. S. Park, D.H. Wang, D. Zhang et al., J. Mater. Sci. Mater. Med., 19, 2317-24 (2008)

8. F.R. Tay, D.H. Pashley, J. Dent., 32(3), 173-96 (2004)

9. R. Wang, Y. Shi, T. Li, Y. Pan, Y. Cui, W. Xia , J. Dent., 62, 72-80 (2017)

10. M.A.M. Proenca, K.T.L. da Silva, A. Costa E. Silva, E.M. Carvalho, J. Bauer, Int. J. Dent., 17(2) (2020)

11. J.H. Jang, M.G. Lee, S.U. Woo, C.O. Lee, J.K. Yi, D.S. Kim, Dent. Mater. J., 35(4), 606-12 (2016)

12. A.M. El Murad, Open Dent. J., 28(12), 664-678 (2018)

13. J.H. Jang, M.G. Lee, S.U. Woo, C.O. Lee, J.K. Yi, D.S. Kim, Dent. Mater. J., 35(4), 606-12 (2016)

14. A.V. Legkikh, J.V. Mandra, Actual problems in Dent., 13(4), 15-21 (2017)

15. V.V. Bazarnyi, L.G. Polushina, A.Yu. Maksimova, E.N. Svetlakova, Yu.V. Mandra, Clin. Lab. Diagn., 12, 773-776 (2018) 